\title{
The Love of Money and Pay Level Satisfaction: Measurement and Functional Equivalence in 29 Geopolitical Entities around the World
}

Thomas Li-Ping Tang, Toto Sutarso, ${ }^{1}$ Adebowale Akande, ${ }^{2}$ Michael W. Allen, ${ }^{3}$ Abdulgawi Salim Alzubaidi, ${ }^{4}$ Mahfooz A. Ansari, ${ }^{5}$ Fernando Arias-Galicia, ${ }^{6}$ Mark G. Borg, ${ }^{7}$ Luigina Canova, ${ }^{8}$ Brigitte Charles-Pauvers, ${ }^{9}$ Bor-Shiuan Cheng, ${ }^{10}$ Randy K. Chiu, ${ }^{11}$ Linzhi Du, ${ }^{12}$ Ilya Garber, ${ }^{13}$ Consuelo Garcia De La Torre, ${ }^{14}$ Rosario Correia Higgs, ${ }^{15}$ Abdul Hamid Safwat Ibrahim, ${ }^{16}$ Chin-Kang Jen, ${ }^{17}$ Ali Mahdi Kazem, ${ }^{18}$ Kilsun Kim, ${ }^{19}$ Vivien Kim Geok Lim, ${ }^{20}$ Roberto Luna-Arocas, ${ }^{21}$ Eva Malovics, ${ }^{22}$ Anna Maria Manganelli, ${ }^{23}$ Alice S. Moreira, ${ }^{24}$ Anthony Ugochukwu Obiajulu Nnedum, ${ }^{25}$ Johnsto E. Osagie, ${ }^{26}$ AAhad M. Osman-Gani, ${ }^{27}$ Francisco Costa Pereira, ${ }^{28}$ Ruja Pholsward, ${ }^{29}$ Horia D. Pitariu, ${ }^{30}$ Marko Polic, ${ }^{31}$ Elisaveta Sardzoska, ${ }^{32}$ Petar Skobic, ${ }^{33}$ Allen F. Stembridge, ${ }^{34}$ Theresa Li-Na Tang, ${ }^{35}$ Thompson Sian Hin Teo, ${ }^{36}$ Marco Tombolani, ${ }^{37}$ Martina Trontelj, ${ }^{38}$ Caroline Urbain ${ }^{39}$ and Peter Vlerick ${ }^{40}$ Middle Tennessee State University, USA; ${ }^{1}$ Middle Tennessee State University, USA; ${ }^{2}$ Intermational Institute of Research, South Africa; ${ }^{3}$ University of Sydney, Australia; ${ }^{4}$ Sultan Qaboos University, Oman; ${ }^{5}$ University of Lethbridge, Canada; ${ }^{6}$ Universidad Autónoma del Estado de Morelos, Mexico; ${ }^{7}$ University of Malta, Malta $;{ }^{8}$ University of Padua, Italy; ${ }^{9}$ University of Nantes, France, ${ }^{10}$ National Taiwan University, Taiwan; ${ }^{11}$ Hong Kong Baptist University, Hong Kong; ${ }^{12}$ Nanjing University, China; ${ }^{13}$ Saratov State Socio-Economic University, Russia; ${ }^{14}$ Technological Institute of Monterrey, Mexico; ${ }^{15}$ Polytechnic Institute of Lisbon - Portugal, Portugal; ${ }^{16}$ Iman University, Saudi Arabia; ${ }^{17}$ National Sun-Yat-Sen University, Taiwan; ${ }^{18}$ Sultan Qaboos University, Oman; ${ }^{19}$ Sogang University, South Korea, ${ }^{20}$ National University of Singapore, Singapore, ${ }^{21}$ University of Valencia, Spain; ${ }^{22}$ University of Szeged, Hungary; ${ }^{23}$ University of Padua, Italy; ${ }^{24}$ Federal University of Para, Brazil; ${ }^{25}$ Nnamdi Azikiwe University, Nigeria; ${ }^{26}$ Florida $A$ \& $M$ University, USA; ${ }^{27}$ Nanyang Technological University, Singapore; ${ }^{28}$ Polytechnic Institute of Lisbon - Portugal, Portugal;

${ }^{29}$ University of the Thai Chamber of Commerce, Thailand; ${ }^{30}$ Babes-Bolyai University, Romania; ${ }^{31}$ University of Ljubljana, Slovenia; ${ }^{32}$ University St. Cyril and Methodius, Macedonia; ${ }^{33}$ Middle Tennessee State University, USA; ${ }^{34}$ Southwestern Adventist University, USA $;{ }^{35}$ Affinion Group, Brentwood, TN, USA; ${ }^{36}$ National University of Singapore, Singapore; ${ }^{37}$ University of Padua, Italy;

${ }^{38}$ University of Ljubljana, Slovenia; ${ }^{39}$ University of Nantes, France; ${ }^{40}$ Ghent University, Belgium 
ABSTRAGT Demonstrating the equivalence of constructs is a key requirement for crosscultural empirical research. The major purpose of this paper is to demonstrate how to assess measurement and functional equivalence or invariance using the 9-item, 3-factor Love of Money Scale (LOMS, a second-order factor model) and the 4-item, 1-factor Pay Level Satisfaction Scale (PLSS, a first-order factor model) across 29 samples in six continents $(\mathrm{N}=5973)$. In step 1, we tested the configural, metric and scalar invariance of the LOMS and 17 samples achieved measurement invariance. In step 2, we applied the same procedures to the PLSS and nine samples achieved measurement invariance. Five samples (Brazil, China, South Africa, Spain and the USA) passed the measurement invariance criteria for both measures. In step 3, we found that for these two measures, common method variance was non-significant. In step 4, we tested the functional equivalence between the Love of Money Scale and Pay Level Satisfaction Scale. We achieved functional equivalence for these two scales in all five samples. The results of this study suggest the critical importance of evaluating and establishing measurement equivalence in cross-cultural studies. Suggestions for remedying measurement nonequivalence are offered.

KEYwORDS the love of money, pay level satisfaction, measurement invariance, functional equivalence, cross-cultural empirical research, 29 geopolitical entities

\section{INTRODUCTION}

Management and organization researchers define measurement as the systematic assignment of numbers on variables to represent characteristics of persons, objects or events (Vandenberg and Lance, 2000). Over the years, management researchers have become increasingly interested in measurement invariance/equivalence (MI/E) due to (i) recent advances in analytic tools and measurement theories and (ii) the importance of valid psychological measurements in cross-cultural studies (Cheung and Rensvold, 2002).

In cross-cultural research, many studies are subject to very severe ethnocentrism (Boyacigiller and Adler, 1991), assuming that measurement scales developed and used in one culture (i.e., the USA) will be universally applicable to other cultures (e.g., China). Moreover, the bulk (64\%) of cross-cultural research in consumer studies covered only two countries and little (23\%) involved more than two countries (Sin et al., 1999). Studies with an insufficient number of cultures (two or three) should be treated only as pilot studies due to their limited usefulness (Samiee and Jeong, 1994). Thus, 'more than two cultures should be used in future research so that findings can be more generalizable' (Sin et al., 1999, p. 89). One of the widely cited cross-cultural studies involving a large number of countries is on the dimensions of national culture (e.g., Hofstede, 1980).

It is premature to test a theoretical relationship between two constructs across cultures 'unless there is confidence that the measures operationalizing the constructs of that relationship exhibit both conceptual and measurement equivalence across the comparison groups' (Riordan and Vandenberg, 1994, p. 645). Without 
construct equivalence, conclusions of studies using a scale developed in one culture to other cultures could all be flawed.

The major purpose of this paper is to illustrate how to assess measurement and functional equivalence using the 9-item, 3-factor Love of Money Scale (LOMS) (e.g., Tang and Chiu, 2003) across 29 geopolitical entities/samples in six continents $(\mathrm{N}=5973)$. In step 1 , we examine measurement invariance of the Love of Money Scale (a second-order factor model) using the most recent measurement theories and techniques (e.g., Chen et al., 2005; Cheung, 2002; Cheung and Rensvold, 2002; Hu and Bentler, 1999; Riordan and Vandenberg, 1994; Vandenberg and Lance, 2000). In step 2, in order to examine functional equivalence of the Love of Money Scale, we select the 4-item, 1-factor Pay Level Satisfaction Scale (PLSS), a subscale of the Pay Satisfaction Questionnaire (PSQ) (e.g., Heneman and Schwab, 1985; Williams et al., 2006) as a criterion and investigate the $\mathrm{MI} / \mathrm{E}$ of the scale following the same procedure in step 1. After we establish measurement invariance for both scales, we then focus on the issue of common method biases in step 3 (Podsakoff et al., 2003). In step 4, we assess functional equivalence by examining the relationship between the love of money and pay level satisfaction.

We select the Love of Money Scale and the Pay Level Satisfaction Scale for the following reasons. First, money is the instrument of commerce and the measure of value (Smith, 1776/1937). For the past several decades, the importance of money has been increasing. For example, only $49.9 \%$ of USA freshmen in 1971 indicated that the important reason in deciding to go on to college is 'to make more money'. In 1993, that number increased to 75.1\% (The American Freshman, 1994). In 1978, men ranked pay the fffth and women ranked pay the seventh in importance, among the ten job preferences in the USA (Jurgensen, 1978). In 1990, among the 11 work goals, pay ranked the second in importance in Belgium, the UK, and the USA and the first in West Germany (Harpaz, 1990). Most Chinese in Hong Kong and China have the cash mentality and prefer cash among 35 components of compensation (Chiu et al., 2001). The lack of money has become the number one cause of dissatisfaction among university students on campuses (out of ten causes) for the most recent period (1997-2003), up from third (1990 96) and second place (1981-87) of two earlier periods (Bryan, 2004). People in the USA and around the world are keenly aware of the importance of money.

Secondly, money has been used to attract, retain and motivate employees and achieve organizational goals in many countries (e.g., Lawler, 1971; Milkovich and Newman, 2005; Tang et al., 2000). Researchers and managers have great interest both in money and in compensation in organizations - pay dissatisfaction has 'numerous undesirable consequences' (Heneman and Judge, 2000, p. 77), such as turnover (Hom and Griffeth, 1995), low commitment, and counterproductive (Cohen-Charash and Spector, 2001) and unethical behaviour (e.g., Chen and Tang, 2006; Tang and Chiu, 2003). 
Thirdly, the meaning of money can be used as the 'frame of reference' (Tang, 1992) in which people examine their everyday lives, such as pay satisfaction (Tang et al., 2005) and life satisfaction (Tang, in press). This leads to the importance of money attitudes. Tang and his associates have developed the Love of Money Scale (LOMS) and examined the love of money with pay satisfaction and other measures in the USA, China, Hong Kong, Spain, Taiwan, the UK and other geopolitical entities (e.g., Du and Tang, 2005; Tang and Chiu, 2003; Tang et al., 2002, 2005). For example, the love of money is directly related to low pay satisfaction among professionals in Hong Kong (Tang and Chiu, 2003), but indirectly related to low pay satisfaction among professors in the USA and Spain (Tang et al., 2005). We, however, cannot take the measurement invariance/ equivalence (MI/E) of the LOMS for granted because it has not been systematically examined across a large number of cultures. This study fills the void in assessing the measurement invariance of this LOMS across a large number of geopolitical entities.

\section{CONCEPTUAL BAGKGROUND AND LITERATURE REVIEW}

There are many measures of attitudes to money in the literature (e.g., Furnham and Argyle, 1998; Opsahl and Dunnette, 1966; Wernimont and Fitzpatrick, 1972). Tang and his associates investigated the meaning of money based on the ABC. model of an attitude with affective, behavioural and cognitive components, and developed several versions of the multidimensional Money Ethic Scale or MES (Tang, 1992; Tang et al., 2000). The LOMS is a subset of the MES (Du and Tang, 2005; Tang and Chiu, 2003). Mitchell and Mickel (1999) considered the MES (Tang, 1992) as one of the most 'well-developed' and systematically used measures of money attitude (Mitchell and Mickel, 1999, p. 571). MES and LOMS have been cited and published in Chinese, English, French, Italian, Spanish, Romanian, Russian and many other languages (see Luna-Arocas and Tang, 2004).

We choose to analyze the 9-item LOMS rather than the entire 58-item MES for three reasons. First, the MES is too long to be practical in a large cross-cultural study. The crux of the matter regarding the meaning of money is the love of it. Thus, we focused on a short, simple, specific and easy-to-use measure. Secondly, in order to decrease the number of indicators used in the model (for parsimony), yet maintain the estimation of measurement error given by multiple-item indicators using structural equation modeling (SEM), researchers must reduce the number of items and constructs to a manageable level. Using parcels (raw item responses combined into subscales) may have detrimental effects on tests of measurement invariance of factor loadings (Bandalos and Finney, 2001). Thirdly, researchers have recognized the importance of the short LOMS in a series of studies, summarized briefly below. 


\section{Past Research on the Love of Money Scale}

Researchers have examined the measurement invariance of the LOMS across gender and college majors (law, sociology and political science) of Chinese students (Du and Tang, 2005), across gender and cultures (the USA vs. Spain) of professors (Tang et al., 2005) and across gender and employment status (full-time vs. parttime) of employees in the USA (Tang, in press). In addition, mental health professionals with a high love of money have high income and high voluntary turnover 18 months later (Tang et al., 2000). The love of money is directly related to unethical behavior or evil (the Love of Money $\rightarrow$ Evil) in a SEM model (Tang and Chiu, 2003). The love of money is negatively related to pay satisfaction (PSQ) that is, in turn, positively related to evil (the Love of Money $\rightarrow$ Pay Satisfaction $\rightarrow$ Evil) (Tang and Chiu, 2003). The unethical behavior or evil construct is a second-order latent factor with several first-order latent constructs: resource abuse, not whistle blowing, theft, corruption, and deception (Tang and Chiu, 2003; Chen and Tang, 2006). This study concerns the relationship between the love of money and pay level satisfaction (the Love of Money $\rightarrow$ Pay Level Satisfaction). In summary, preliminary evidence suggests that the LOMS is a useful measure for cross-cultural research. The current study engages in a formal examination of the measurement invariance of this scale across many geopolitical entities.

\section{What is the Love of Money?}

The first question a scientific investigator must ask is not 'How can I measure it?' but rather, 'What is it?' (Locke, 1969, p. 334). We trace the inspiration to study the love of money construct to the oldest references in the literature: 'Poverty consists, not in the decrease of one's possessions, but in the increase of one's greed' (Plato, 427-347 BC). 'People who want to get rich fall into temptation and a trap and into many foolish and harmful desires that plunge men into ruin and destruction. For the love of money is a root of all kinds of evil' (http://www.biblegateway.com, 1 Timothy, 6: 9-10, New International Version). 'Whoever loves money never has money enough; whoever loves wealth is never satisfied with his income' (http:// www.biblegateway.com, Ecclesiastes, 5: 10, New International Version). Thus, 'wanting to be rich' may be related to 'the love of money' that may in turn be related to low pay satisfaction.

Researchers (e.g., Tang and Chiu, 2003) have offered various definitions of the love of money. It is: (i) one's attitudes towards money; (ii) one's meaning of money; and (iii) one's wants, desires, values and aspirations of money (Tang, in press), but it is not one's needs, greed or materialism (Belk, 1985). It is a multidimensional individual difference variable with affective, behavioural and cognitive components (Tang, 1992). There are three types of multidimensional constructs: the latent model, the aggregate model and the profile model (Law et al., 1998). We 


\section{The Love of Money}

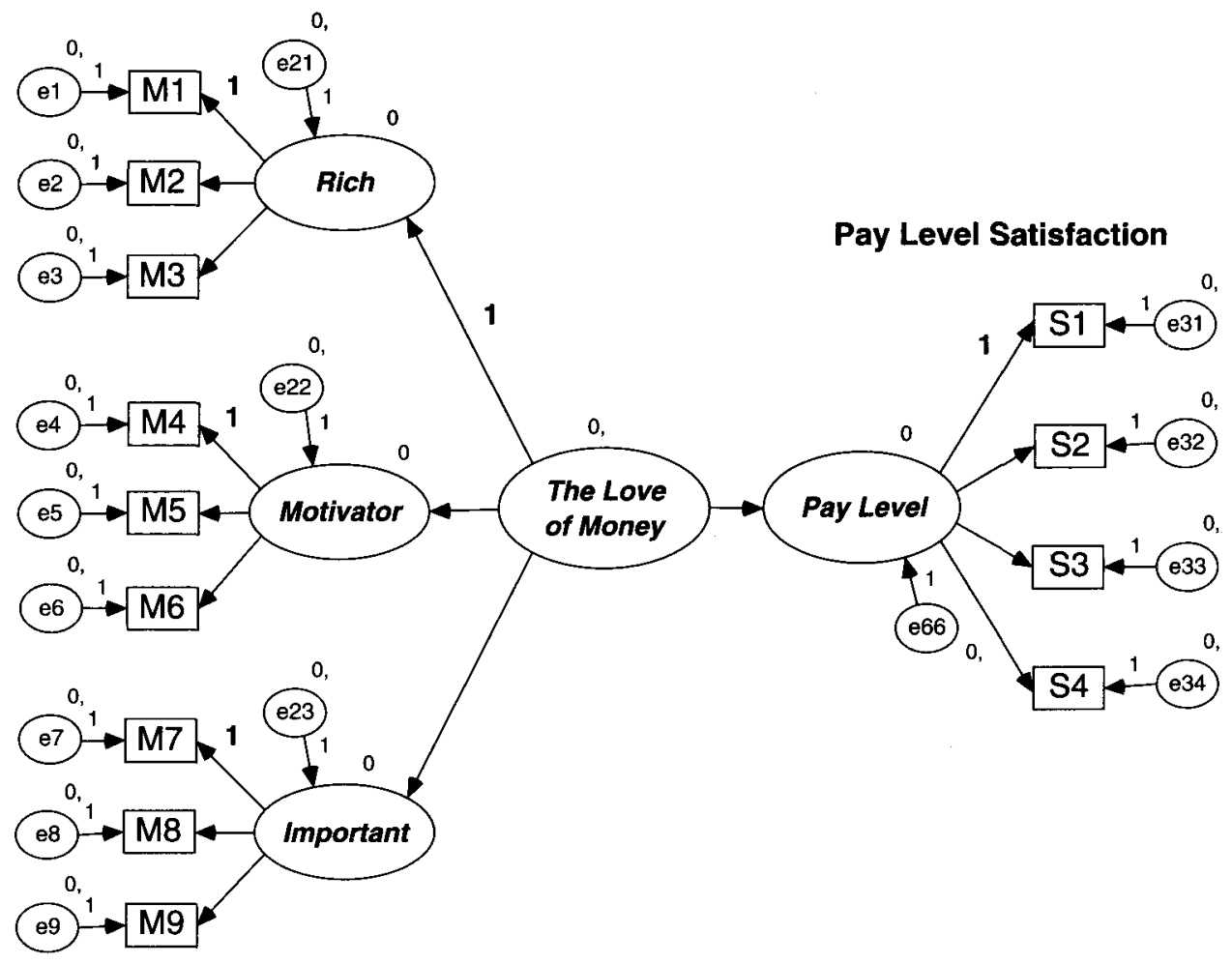

Figure 1. A model of the love of money and pay level satisfaction

adopted the latent model to define the love of money construct in this study. The love of money is an unobservable second-order latent construct that has three first-order latent constructs: rich, motivator, and important. Each first-order latent construct is measured by three observable items (see the left side of Figure 1 and Appendix I). Specifically, we argue that if one has a high level of love of money, one may: (i) have a high desire to be rich (affective); (ii) be highly motivated by money (behavioural); and (iii) consider money as a very important part of one's life (cognitive). We defined these first-order factors below.

Rich. The affective component of love of money refers to one's love or hate orientation, feeling or emotion regarding money. Do you love or hate money? Is money good or evil (Tang, 1992)? We speculate that most people love money and very few hate money. If one loves money, one wants to have a lot of it. This leads to one's desire to get rich. Being rich is good and is better than being poor; thus most people want to be rich. Research suggests that children from poor economic backgrounds tend to overestimate the size of a coin and place greater 
importance on money than those from rich families (Bruner and Goodman, 1947). People who have experienced financial hardship tend to be obsessed with money (Lim and Teo, 1997). Past research using confirmatory factor analysis (CFA) shows that factor rich has the highest factor loading of the three factors, for the love of money construct (Tang and Chiu, 2003). Thus, a large part of the common variance of the love of money construct comes from factor rich (cf. Law et al., 1998).

Motivator. This behavioural component refers to how one intends or expects to act towards someone or something. In the case of money, one may consider how one makes money, how one budgets one's money, how one spends one's money, and how one contributes to church, charity and society (e.g., Furnham and Argyle, 1998; Tang, 1992). Money is a motivator for some (e.g., Harpaz, 1990; Kohn, 1993; Stajkovic and Luthans, 2001), but not for others (e.g., Herzberg, 1987; Pfeffer, 1998). If one has a high love of money, one will be highly motivated by money, will work hard for money and will take actions and do whatever it takes to make money. Regarding improving performance in organizations, 'no other incentive or motivational technique comes even close to money' (Locke et al., 1980 , p. 381). In response to a bonus plan that paid people for finding insect parts in a food process plant, innovative employees 'brought insect parts from home to add to the peas just before they removed them and collected the bonus' (Milkovich and Newman, 2005, p. x). Love of money may motivate people to take actions involving even unethical behaviour.

Important. The cognitive component of money refers to important beliefs or ideas one has about money. For example, money means power, freedom, respect, security, etc. (e.g., Furnham and Argyle, 1998; Tang, 1992). This study focuses on only one cognitive component: money is important. If one has a high level of the love of money, one will consider money as one of the most important parts of one's life. The most consistent thread of the money attitude literature is the 'emphasis on its importance' (Mitchell and Mickel, 1999, p. 569). The importance of money is formed early in childhood and maintained in adult life (Furnham and Argyle, 1998). These three first-order factors contribute to the love of money that may lead to low pay satisfaction in organizations (Tang and Chiu, 2003).

\section{Pay Satisfaction}

Job satisfaction may be defined as 'a pleasurable or positive emotional state resulting from the appraisal of one's job or job experiences' (Locke, 1976, p. 1300). Pay satisfaction is a part of job satisfaction. The two most widely known and used models of pay satisfaction are the equity model and the discrepancy model (Heneman and Judge, 2000). The equity model of pay satisfaction depends on the 
comparison of the person's outcome-input ratio to the outcome-input ratio of a comparison other (Adams, 1963). The pay discrepancy model focuses on the difference between 'expectation' and 'reality' in pay (Rice et al., 1990). 'The consistency of the pay level-pay satisfaction relationship is probably the most robust (though hardly surprising) finding regarding the causes of pay satisfaction' (Heneman and Judge, 2000, p. 71). Actual pay level (income) is consistently and positively related to pay satisfaction.

In order to examine functional equivalence for the LOMS, we need to select a short and easy to use criterion. The 18-item, 4-factor Pay Satisfaction Questionnaire (PSQ Heneman and Schwab, 1985) is one of the most well-known multidimensional measures of pay satisfaction (e.g., Williams et al., 2006). We used the 4-item pay level subscale of the PSQ labeled it as Pay Level Satisfaction Scale (PLSS) in this study (see the right-hand side of Figure 1 and Appendix I), and related it to the LOMS.

\section{The Love of Money to Pay Level Satisfaction Relationship}

The love of money reflects individuals' frames of reference regarding values, standards, expectations, or aspirations of pay and is used in judging pay satisfaction. If money is important to them, they may pay more attention to and are constantly aware of others' pay in the society. If one has a high love of money, one expects to have a large output (pay) for one's work (the equity theory), or high expectation for one's pay (the discrepancy theory). This leads to a lower output/ input ratio compared with the referents or a large gap between expectation and reality. The Chinese expression of 'The raising tides lift all boats (水涨船高)' implies that when one's income increases, one raises the standard. The more money someone has, the more they want it. The love of money may increase accordingly, up to a point. Most people compare themselves with the rich. When they compare themselves with the rich, they get upset and angry, that is, a sense of relative deprivation (Vanneman and Pettigrew, 1972) which leads to low pay satisfaction. These theories predict that those with a high love of money may have low pay level satisfaction. The purpose of this study is not to establish the substantive relationship between these two constructs per se but to provide a baseline prediction in order to examine functional equivalence across cultures. This is a good example since it is unclear if the negative relationship observed thus far exists in all cultures.

\section{Measurement Invariance}

There are nine steps of measurement invariance: (i) an omnibus test of equality of covariance matrices across groups; (ii) a test of configural invariance; (iii) a test of metric invariance; (iv) a test of scalar invariance; (v) a test of the null hypothesis that like items unique variances are invariant across groups; (vi) a test of the null 
hypothesis that factor variances are invariant across groups; (vii) a test of the null hypothesis that factor covariances are invariant across groups; (viii) a test of the null hypothesis of invariant factor means across groups; and (ix) other more specific tests. Among these nine steps, 'tests for configural and metric invariance were most often reported' (Vandenberg and Lance, 2000, p. 35, emphasis added). Category 1 invariance is related to the psychometric properties of the measurement scales (configural, metric and scalar invariance) and category 2 invariance is associated with between-group differences (latent means, variances and covariances). The category 1 invariance is a prerequisite for the interpretation of category 2 differences, where category 2 differences involve substantive research interests to scholars (Cheung and Rensvold, 2002). The present study deals with some of these issues.

The LOMS fits the second-order factor model (Fig. l) because the three lower order factors (rich, motivator, and important) are substantially correlated with each other and there is a higher order factor (the love of money) that is hypothesized to account for the relations among the lower order factors. In this study, we follow suggestions in the literature (e.g., Chen et al., 2005; Cheung and Rensvold, 2002; Riordan and Vandenberg, 1994; Vandenberg and Lance, 2000) and investigate: (i) configural (factor structures) invariance; (ii) the first-order metric (factor loading) invariance; (iii) item-level metric invariance; (iv) scalar (intercepts of measured variables) invariance; (v) first-order latent mean comparison; (vi) second-order metric invariance; (vii) second-order scalar invariance; and (viii) second-order latent mean comparison for the LOMS (the second-order factor model) and the first five steps for the PLSS (the first-order factor model). Configural invariance refers to the equality of factor structures or equal number of factors and factor patterns. The same item must be an indicator of the same latent factor across groups. Researchers use CFA to examine the invariance of measurement form (factor structures) for each group. Metric invariance is achieved when the differences between the unconstrained and the constrained (all factor-loading parameters are set to be equal) multigroup confirmatory factor analyses (MGCFAs) are non-significant. Thus, the unit of the measurement of the underlying factor is identical across samples. Scalar (intercept) invariance is achieved when the origin of the scale is the same across groups. This is required for comparing latent mean differences across samples. This is an important and crucial part of cross-cultural studies since it gives us information on whether or not groups have similar mean scores on a construct due to measurement.

\section{Common Method Biases}

Cross-sectional data with mono-method and mono-source may create additional method biases (one of the main sources of measurement errors) that may pose a major threat to the validity of the conclusion about the relationship between 
measures (Podsakoff et al., 2003). If the measures of construct A and the measures of construct $B$ share common methods, then these methods may exert a systematic effect (inflate, deflate or have no effect) on the observed relationship between these two measures. About one quarter $(26.3 \%)$ of the variance in a typical research measure might be due to systematic sources of measurement errors such as common method biases. Attitude measures, in particular, may contain an average of $40.7 \%$. Podsakoff et al. (2003) offered a complete review of all sources of common method variance and procedural and statistical remedies for controlling common method biases. In this study, (i) we employ Harman's single-factor test (EFA) and (ii) we control for the effects of a single unmeasured latent method factor (CFA) in our analyses.

\section{METHOD}

\section{Sample}

The first author recruited researchers in approximately 50 geopolitical entities through personal friends, contacts, or networking at professional conferences of the Academy of Management, Academy of Human Resource Development, International Association for Research in Economic Psychology, International Association of Applied Psychology and Society for Industrial and Organizational Psychology. Researchers received a 19-page package including a six-page survey (informed consent and items) and instructions (references, websites, translation procedures). $\mathrm{He}$ asked collaborators to collect data from at least 200 full-time white-collar employees or managers in large organizations. The dataset for this paper is a part of a larger cross-cultural study.

We received 31 samples from 30 geopolitical entities $(N=6659)$ in the period of December 2002 to January 2005. We selected 29 samples of full-time employees $(\mathrm{N}=5973)$ and eliminated a duplicate sample from Singapore and a student sample from China. Our convenience samples may not represent the whole population or the average citizens of the geopolitical entities. On average, participants in this study were 34.70 years old $(\mathrm{SD}=9.92)$ with $50 \%$ male and had 15.46 years of education ( $\mathrm{SD}=3.26$ ). Table 1 shows the sample size, the basic demographic information and the means and standard deviations of the two measures for each of these 29 samples.

\section{Measures}

Researchers in each geopolitical entity organized small focus groups and translated the English version to their own native languages using a multi-stage translation-back-translation procedure (Brislin, 1980). We used 5-point Likerttype scales. The response scale anchors for the 9-item LOMS were: strongly 
The Love of Money

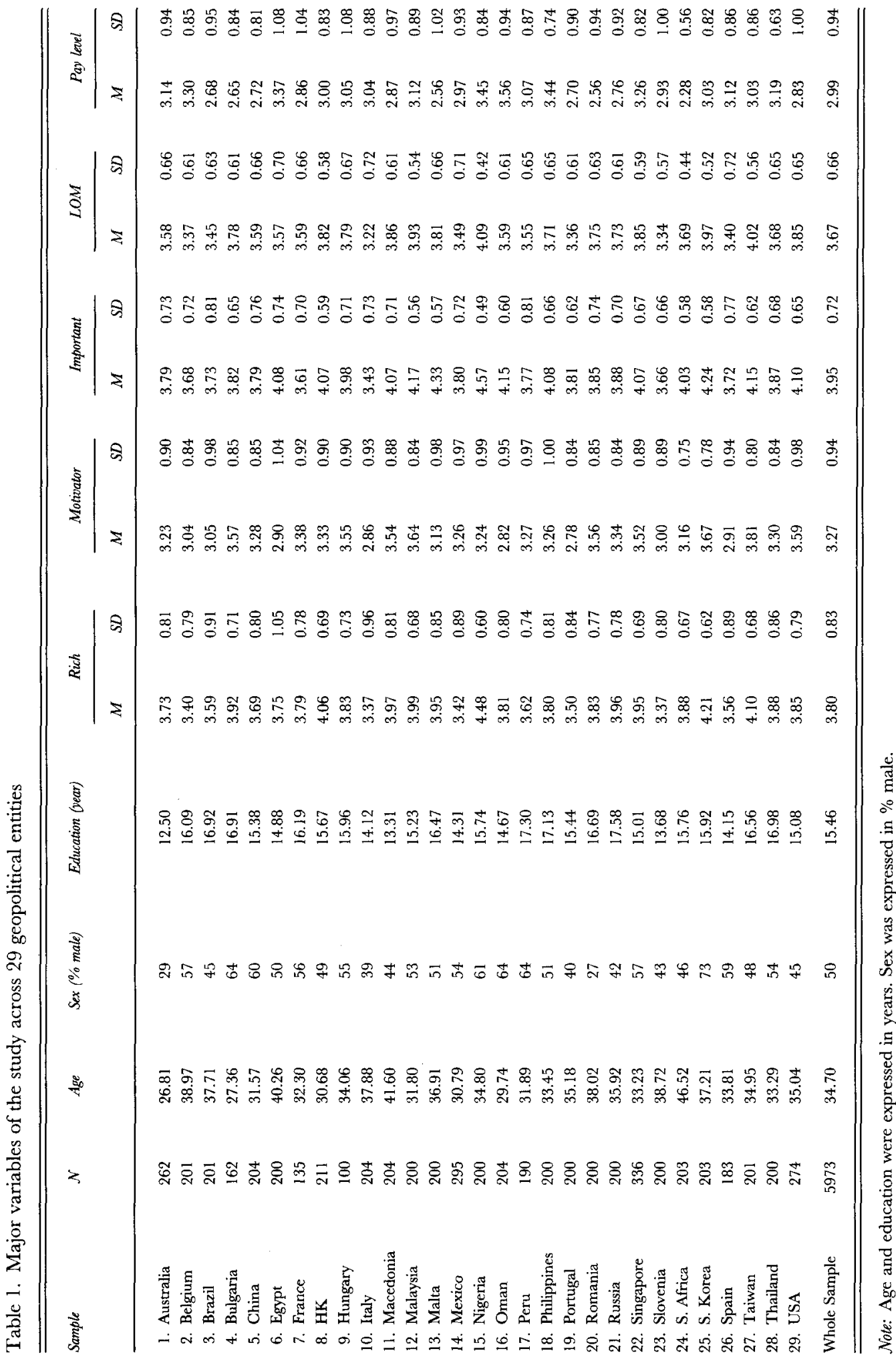


disagree (1); neutral (3); and strongly agree (5). For the 4-item PLSS, the response anchors were: strongly dissatisfied (1); neutral (3); and strongly satisfied (5). Participants completed the survey voluntarily and anonymously. The reliability (Cronbach's alpha) for the total sample was 0.85 (LOMS) and 0.90 (PLSS), respectively.

\section{Evaluation Criteria for Measurement Invariance}

Researchers have recommended several criteria for evaluating configural invariance: (i) $\chi^{2}$, df, and $\mathrm{p}$ value; (ii) $\chi^{2} / \mathrm{df}<3$; (iii) Tucker-Lewis Index, TLI $>0.95$; (iv) relative noncentrality index, RNI $>0.95$; (v) comparative fit index, CFI $>0.95$; (vi) the standardized root mean square residual, SRMSR $<0.08$; and (vii) root mean square error of approximation, RMSEA $<0.08$ (Vandenberg and Lance, 2000). A lower value of $\chi^{2}$ indicates a better fit and it should be non-significant. However, for large sample sizes, this statistic may lead to rejection of a model with good fit. Given these problems with the $\chi^{2}$, we used the following four rigorous evaluation criteria, TLI $>0.95$, GFI $>0.95$, SRMSR $<0.08$, and RMSEA $<0.08$, even though we report the $\chi^{2}$ values for reference. The evaluation criteria for metric invariance include the change of $\chi^{2}$ relative to the change of degree of freedom between the unconstrained and the constrained MGCFA and associated change in CFI. Changes in $\chi^{2}$ are sensitive to sample size; and because of the large sample size in multiple sample SEMs, almost any trivial noninvariance will result in significant changes in $\chi^{2}$ if equality constraints are added. Cheung and Rensvold (2002) recommend using changes in CFI $(<0.01)$ as a rule of thumb (i.e., if $\Delta \mathrm{CFI}=0.01$ or less: differences between models do not exist). We apply this criterion when we investigate metric invariance and functional equivalence.

\section{RESULTS}

\section{Step 1: Measurement Invariance of the Love of Money Scale}

Model 1: Configural (factor structures) invariance. We examined the fit between the 9-item, 3-factor love of money measurement model and data from each sample and repeated the procedure 29 times (Table 2). On the basis of four rigorous criteria, we eliminated 12 samples and retained 17 samples in this analysis. If configural invariance is not demonstrated across groups, further tests are then unwarranted (Vandenberg and Lance, 2000).

To identify the possible reasons for the non-invariance in a sample, we used exploratory factor analysis (EFA). For example, for the sample from Malta, item 3 (see Appendix I) was related to both factors rich (0.86) and important (0.42); item 6 was strongly related to both factors motivator $(0.76)$ and rich $(0.46)$; and item 9 
Table 2. Configural invariance of the 9-item, 3-factor Love of Money Scale (LOMS)

\begin{tabular}{|c|c|c|c|c|c|c|c|}
\hline & $\chi^{2}$ & $d f$ & $p$ & $T L I$ & $C F I$ & $S R M S R$ & RMSEA \\
\hline 1. Australia & 74.47 & 24 & 0.00 & 0.9874 & 0.9933 & 0.0561 & 0.0898 \\
\hline 2. Belgium & 27.41 & 24 & 0.29 & 0.9988 & 0.9994 & 0.0416 & 0.0266 \\
\hline 3. Brazil & 26.49 & 24 & 0.33 & 0.9992 & 0.9996 & 0.0412 & 0.0228 \\
\hline 4. Bulgaria & 34.37 & 24 & 0.08 & 0.9973 & 0.9986 & 0.0386 & 0.0428 \\
\hline 5. China & 34.82 & 24 & 0.07 & 0.9965 & 0.9981 & 0.0337 & 0.0471 \\
\hline 6. Egypt & 29.64 & 24 & 0.20 & 0.9979 & 0.9989 & 0.0369 & 0.0344 \\
\hline 7. France & 37.98 & 24 & 0.03 & 0.9929 & 0.9962 & 0.0480 & 0.0659 \\
\hline 8. HK & 46.43 & 24 & 0.00 & 0.9939 & 0.9968 & 0.0437 & 0.0667 \\
\hline 9. Hungary & 107.09 & 24 & 0.00 & 0.9501 & 0.9734 & 0.0760 & 0.1870 \\
\hline 10. Italy & 51.98 & 24 & 0.00 & 0.9905 & 0.9950 & 0.0424 & 0.0758 \\
\hline 11. Macedonia & 60.84 & 24 & 0.00 & 0.9885 & 0.9939 & 0.0518 & 0.0870 \\
\hline 12. Malaysia & 106.90 & 24 & 0.00 & 0.9772 & 0.9879 & 0.0520 & 0.1317 \\
\hline 13. Malta & 445.66 & 24 & 0.00 & 0.8931 & 0.9430 & 0.1197 & 0.2971 \\
\hline 14. Mexico & 79.35 & 24 & 0.00 & 0.9873 & 0.9932 & 0.0506 & 0.0886 \\
\hline 15. Nigeria & 92.67 & 24 & 0.00 & 0.9802 & 0.9938 & 0.1201 & 0.1228 \\
\hline 16. Oman & 15.26 & 24 & 0.91 & 1.0000 & 1.0000 & 0.0255 & 0.0000 \\
\hline 17. Peru & 60.03 & 24 & 0.00 & 0.9881 & 0.9937 & 0.0485 & 0.0891 \\
\hline 18. Philippines & 73.16 & 24 & 0.00 & 0.9852 & 0.9921 & 0.0477 & 0.1015 \\
\hline 19. Portugal & 30.39 & 24 & 0.17 & 0.9979 & 0.9989 & 0.0345 & 0.0366 \\
\hline 20. Romania & 60.24 & 24 & 0.00 & 0.9883 & 0.9938 & 0.0471 & 0.0871 \\
\hline 21. Russia & 33.59 & 24 & 0.09 & 0.9969 & 0.9983 & 0.0356 & 0.0448 \\
\hline 22. Singapore & 95.95 & 24 & 0.00 & 0.9877 & 0.9934 & 0.0454 & 0.0946 \\
\hline 23. Slovenia & 41.30 & 24 & 0.02 & 0.9940 & 0.9968 & 0.0593 & 0.0602 \\
\hline 24. S. Africa & 37.64 & 24 & 0.04 & 0.9948 & 0.9973 & 0.0582 & 0.0530 \\
\hline 25. S. Korea & 43.74 & 24 & 0.01 & 0.9951 & 0.9974 & 0.0415 & 0.0638 \\
\hline 26. Spain & 41.08 & 24 & 0.02 & 0.9936 & 0.9966 & 0.0463 & 0.0625 \\
\hline 27. Taiwan & 72.01 & 24 & 0.00 & 0.9874 & 0.9933 & 0.0450 & 0.1000 \\
\hline 28. Thailand & 30.64 & 24 & 0.16 & 0.9980 & 0.9989 & 0.0284 & 0.0373 \\
\hline 29. USA & 56.46 & 24 & 0.00 & 0.9927 & 0.9961 & 0.0427 & 0.0704 \\
\hline
\end{tabular}

Note: We retained a sample if it satisfied all of the following four rigorous criteria (i.e. TLI $>0.95$, CFI $>0.95$, SRMSR < 0.08, and RMSEA < 0.08). In this analysis, we eliminated 12 samples (printed in bold) and retained 17 samples.

was strongly associated with factors important (0.76) and rich (0.43). For the Nigerian sample, item 6 was negatively related to factor important $(-0.40)$ and was not related to factor motivator that had only two items. For people in these two samples, their data did not fit our theoretical measurement model of the 9-item, 3-factor LOMS. The aforementioned results are the possible reasons for the non-invariance.

Model 2: Construct-level metric (factor loadings) invariance. We used the 17 samples $(\mathcal{N}=3385)$ that passed the configural invariance test and applied the multiple-group confirmatory factor analyses (MGCFAs) in subsequent tests. For the unconstrained model, we did not put any constrains (Table 3, step 1, 


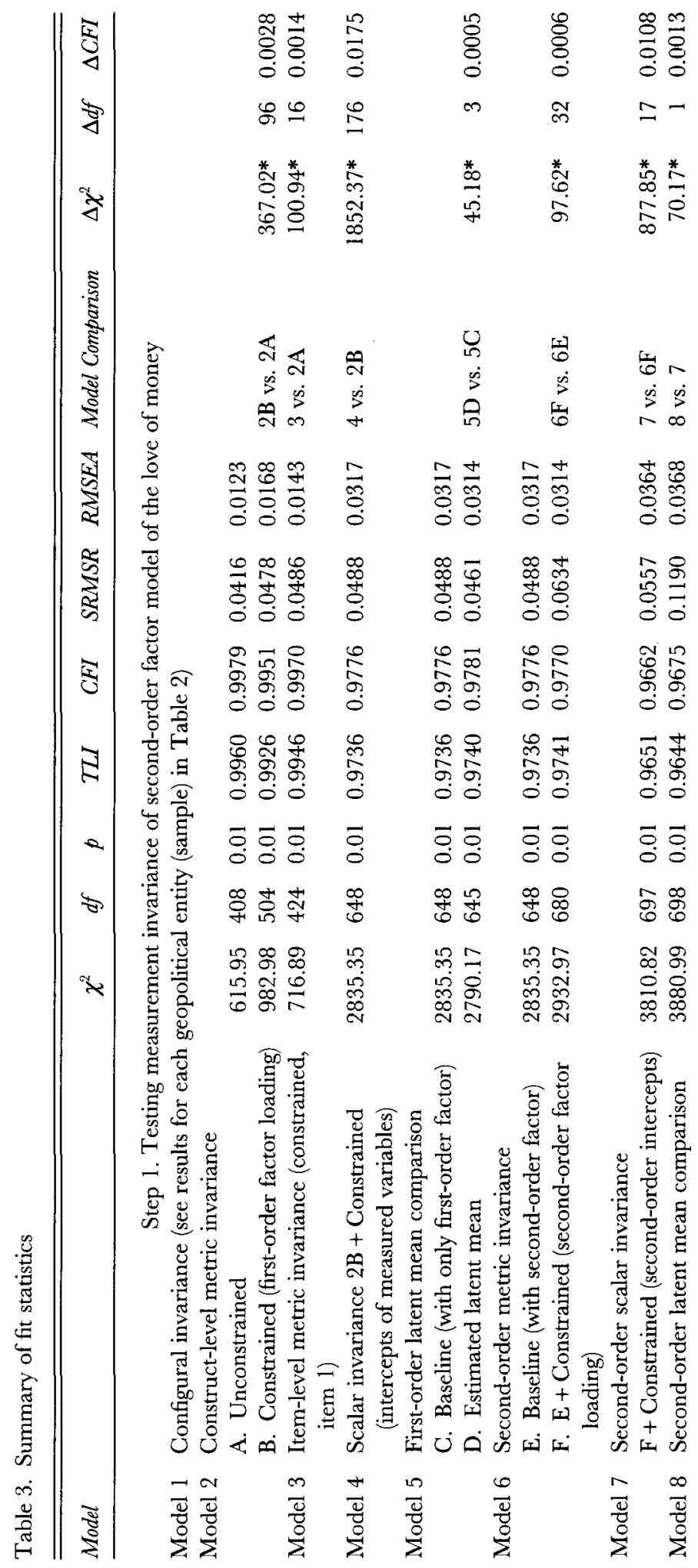


The Love of Money

\begin{tabular}{|c|c|c|c|c|}
\hline $\begin{array}{l}\infty \\
\stackrel{0}{8} \\
\stackrel{0}{\circ}\end{array}$ & $\frac{\mathscr{g}}{\sigma}$ & $\begin{array}{l}\infty \\
\\
0\end{array}$ & $\stackrel{8}{8}$ & 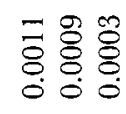 \\
\hline$\Delta^{\infty}$ & के & - & $m$ & $\Sigma \simeq$ \\
\hline & 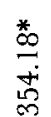 & $\begin{array}{l}\stackrel{*}{*} \\
\stackrel{\sigma}{\sigma} \\
\stackrel{N}{F}\end{array}$ & $\begin{array}{l}* \\
\infty \\
0 \\
0 \\
\infty\end{array}$ & 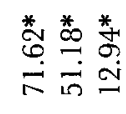 \\
\hline
\end{tabular}

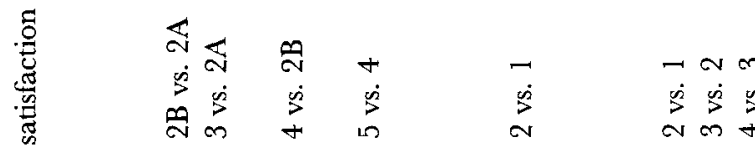

㟧

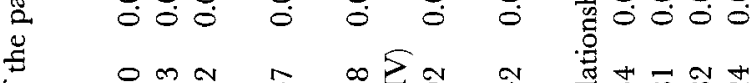

पे

莎

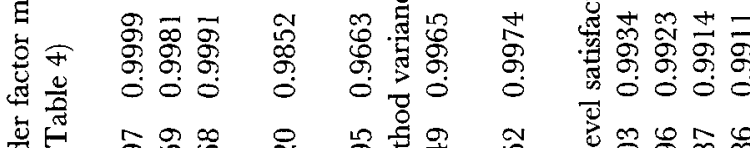

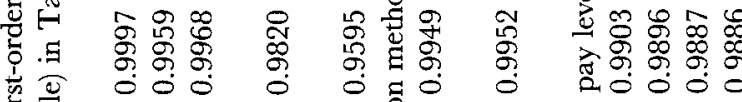

4

$$
\text { 造 }
$$

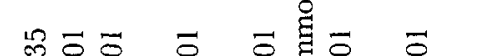

๗융용

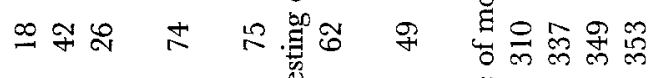

.

苞泀

$\sin$

남 Jै

बळi

吾

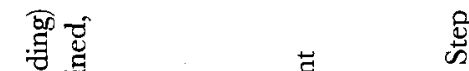
品

要

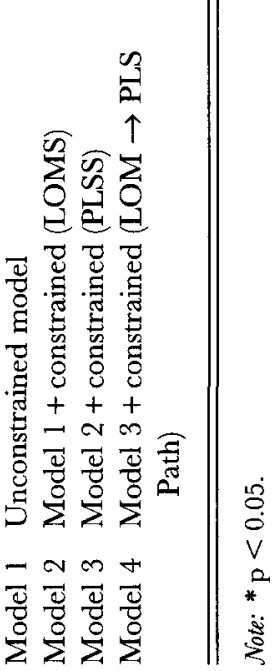


model 2A); for the constrained model, we constrained the first-order factor loadings to be the same across groups (model $2 \mathrm{~B})$. We compared an unconstrained MGCFA model $\left(\chi^{2}=615.95, d f=408, \mathrm{p}<0.01\right.$, TLI $=0.9960, \mathrm{CFI}=0.9979$, SRMSR $=0.0416$, RMSEA $=0.0123)$ with a constrained MGCFA model $\left(\chi^{2}=982.98, d f=504, \mathrm{p}<0.01, \mathrm{TLI}=0.9926, \mathrm{CFI}=0.9951, \mathrm{SRMSR}=0.0478\right.$, RMSEA $=0.0168)$. Due to non-significant fit index change $(\Delta C F I=0.9979-$ $0.9951=0.0028$ ), we concluded that metric equivalence was achieved across the 17 samples for the LOMS (Cheung and Rensvold, 2002).

Model 3: Item-level metric invariance. Results of model 2 indicated that the analyses for model 3 were unnecessary. However, in the spirit of providing useful guidance to future researchers in cross-cultural research, we followed the suggestions in the literature (e.g., Cheung and Rensvold, 2002) and demonstrated additional procedures for identifying the potential sources of metric non-invariance across samples. For example, which 'factor' of the 9-item, 3-factor LOMS could be the major source of non-invariance? After we have identified the factor, which 'item' within the factor could be the major source of non-invariance? After we have identified the item, which 'samples' (geopolitical entities) could be the sources of non-invariance? We list these steps below.

We compared the results of the unconstrained 17-country MGCFA with three separate partially constrained 17-country MGCFAs. In a partially constrained model, we set all (first-order) factor loadings to be equal for one factor while allowing the other two factors to vary. We did this for each first-order factor. We compared the unconstrained model (Table 3, model 2A) with three constrained models: (i) factor rich constrained $\left(\chi^{2}=807.48\right.$, df $=440, \mathrm{p}<0.01$, $\mathrm{TLI}=0.9935, \mathrm{CFI}=0.9962, \mathrm{SRMSR}=0.0467$, RMSEA $=0.0157$ ); (ii) factor motivator constrained $\left(\chi^{2}=663.82, \mathrm{df}=440, \mathrm{p}<0.01, \mathrm{TLI}=0.9960, \mathrm{CFI}=\right.$ 0.9977 , SRMSR $=0.0417$, RMSEA $=0.0123$ ); and (iii) factor important constrained $\left(\chi^{2}=747.60, \mathrm{df}=440, \mathrm{p}<0.01, \mathrm{TLI}=0.9945, \mathrm{CFI}=0.9969, \mathrm{SRMSR}=\right.$ 0.0428$, RMSEA $=0.0144)$. We achieved metric invariance at the factor level based on non-significant fit index change: factor rich $(\Delta \mathrm{CFI}=0.0017)$, factor motivator $(\Delta \mathrm{CFI}=0.0002)$, and factor important $(\Delta \mathrm{CFI}=0.0010)$, respectively (Cheung and Rensvold, 2002). It should be noted that factor rich had the largest CFI change.

Next, we examined partial metric invariance at the 'item' level for all three items of factor rich using the exact same method mentioned above. We achieved metric invariance at the item level for Item 1 because the CFI change was again negligible $(\Delta \mathrm{CFI}=0.0014)$ (Table 3, model 3). It should be noted, however, that item 1 had the largest CFI change.

The $Z$ test can be used to determine the significant difference of parameter estimates between samples. When comparing factor loading across groups, the $Z$ statistic is defined as 


$$
\frac{\hat{\lambda}_{i}^{(1)}-\hat{\lambda}_{i}^{(2)}}{\sqrt{S_{\hat{\lambda}_{i}^{(1)}}^{2}+S_{\hat{\lambda}_{i}^{(2)}}^{2}}}
$$

where the factor loadings are estimates in the unconstrained model and the parenthetical number in superscript denotes the group or sample number (Cheung, 2002). The above formula gives an approximation of the $\chi^{2}$ difference test.

When we examined the factor loadings of item 1 (I want to be rich), we could select either item 2 or item 3 as the marker item. What is invariance with respect to one marker item may be non-invariance with respect to another marker item. To simplify the procedure, we used only item 2 as the marker item. For the 9-item, 3-factor model across 17 samples, we calculated 136 pair-wise comparisons (i.e., $\mathbf{n}(\mathbf{n}-1) / 2, \mathbf{n}=$ the number of samples) for each item and 408 pair-wise tests for all 3 items of factor 1 (136 pair-wise tests $\times 3$ items). To obtain a balance between Type I and Type II errors, we adopted the alpha value of 0.00012 (alpha $=0.05 / 408$ ) for each pair-wise comparison. This translated into a (two-tail) critical $Z$ value of 3.85 (http://math.uc.edu/ brycw/classes/148/ tables.htm). By using a spreadsheet, we input the factor loading parameter estimates (Appendix II, row 1, L), standard errors (row 2, S) of the unconstrained model of item 1 across 17 samples, applied the formula (1) above, and found no significant $Z$ test results. These findings further confirmed our analyses in model 2 that we achieved full metric invariance.

Model 4: Scalar(intercept) invariance. We used model 2B as the foundation and set the intercepts of measured variables to be equal across 17 geopolitical entities and compared the results (model 4) with model 2B. The change of CFI ( $\Delta \mathrm{CFI}=0.0175)$ was greater than 0.01 . When the differences lie between 0.01 and 0.02 , then researchers should be suspicious that differences may exist (Cheung and Rensvold, 2002; Vandenberg and Lance, 2000).

Following the exact procedures of model 3 above, we compared the results of the unconstrained 17-country MGCFA with three separate partially constrained 17-country MGCFAs. In a partially constrained model, we set all intercepts of measured variables to be equal for one (first-order) factor while allowing the other two factors to vary and repeated the same process for each of the three factors. We compared the unconstrained model (Table 3, Model 2B, CFI $=0.9951$ ) with three constrained models: (i) factor rich constrained $\left(\chi^{2}=1801.96, \mathrm{df}=552, \mathrm{p}<0.01\right.$, $\mathrm{TLI}=0.9823, \mathrm{CFI}=0.9872, \mathrm{SRMSR}=0.0444, \mathrm{RMSEA}=0.0259$ ); (ii) factor motivator constrained $\left(\chi^{2}=1505.57, \mathrm{df}=552, \mathrm{p}<0.01, \mathrm{TLI}=0.9865, \mathrm{CFI}=\right.$ 0.9903, $\mathrm{SRMSR}=0.0476, \mathrm{RMSEA}=0.0226$ ); and (iii) factor important constrained $\left(\chi^{2}=1683.24, \mathrm{df}=552, \mathrm{p}<0.01, \mathrm{TLI}=0.9840, \mathrm{CFI}=0.9884\right.$, SRMSR $=0.0478, \mathrm{RMSEA}=0.0247)$. The change of CFI was non-significant for 
factor rich $(\Delta \mathrm{CFI}=0.0079)$, factor motivator $(\Delta \mathrm{CFI}=0.0048)$, and factor important $(\Delta \mathrm{CFI}=0.0067)$, respectively. We achieved full scalar invariance across 17 geopolitical entities and stopped our analysis. If any of the CFI changes were significant, researchers then may identify the non-invariant item(s) and specific samples causing the non-invariance (see model 3).

Model 5: First-order latent mean comparison. We deleted the second-order latent factor (the love of money) and set the three first-order factors (rich, motivator, and important) to be correlated (covariance). This was the baseline model (see Table 3 , model 5C). Using the baseline model, we then estimated latent mean for the three first-order factors (model 5D). To estimate the difference between the factor means, one group is usually chosen as a reference or baseline group (i.e., the first geopolitical entity) and its latent means are set to zero. The latent means of the other 16 groups are estimated. When we compared model $5 \mathrm{D}$ with the baseline model $5 \mathrm{C}$, the change of CFI was negligible $(\Delta \mathrm{CFI}=0.0005)$. Thus, it is appropriate to compare mean differences across geopolitical entities.

Model 6: Second-order metric invariance. We returned to the original model (model 4) as our baseline model (models 4 and $6 \mathrm{E}$ were the same). Using the baseline model, we set the second-order factor loadings to be the same across 17 samples (model 6F). We achieved second-order metric invariance comparing models $6 \mathrm{~F}$ and $6 \mathrm{E}$ due to negligible CFI change (0.0006).

Model 7: Second-order scalar invariance. Using model 6F as the foundation, we set the second-order intercepts to be equal across 17 samples. When we compared the two models ( 7 and $6 \mathrm{~F}$ ), the CFI change for the second-order scalar invariance $(\triangle \mathrm{CFI}=0.0108)$ was greater than 0.01 . It should be pointed out that this CFI change $(0.0108)$ was smaller than that in model $4(0.0175)$. We followed the procedures mentioned in models 3 and 4 and investigated the potential sources of second-order scalar non-invariance across samples. Again, the results were negligible. We achieved second-order scalar invariance.

Model 8: Second-order latent mean comparison. In this analysis, we used model 7 as the foundation and then estimated latent mean for the second-order factor (model 8). To estimate the difference between the factor means, we again used the procedure in model 5, set latent mean of the first group to zero, and set the latent means of the other 16 groups to be estimated. The CFI change (model 8 [constrained means] vs. model 7) was negligible (0.0013). It is appropriate to compare mean differences across samples.

In summary, we apply the most rigorous criteria and achieve measurement invariance for the 9-item, 3-factor LOMS, meaning that the form, unit, origin and latent mean of the scale are the same across 17 geopolitical entities. The non-significant and negligible differences across samples could be mainly related to factor rich. Next, we turn to the measurement invariance of the PLSS. 
Table 4. Configural invariance of the 4-item, 1-factor Pay Level Satisfaction Scale (PLSS)

\begin{tabular}{|c|c|c|c|c|c|c|c|}
\hline & $\chi^{2}$ & $d f$ & $p$ & $T L I$ & $C F I$ & $S R M S R$ & RMSEA \\
\hline 1. Australia & 0.31 & 2 & 0.86 & 1.0000 & 1.0000 & 0.0030 & 0.0000 \\
\hline 2. Belgium & 4.82 & 2 & 0.00 & 0.9951 & 0.9990 & 0.0090 & 0.0839 \\
\hline 3. Brazil & 2.25 & 2 & 0.33 & 0.9994 & 0.9999 & 0.0104 & 0.0250 \\
\hline 4. Bulgaria & 13.35 & 2 & 0.00 & 0.9697 & 0.9939 & 0.0233 & 0.1878 \\
\hline 5. China & 2.84 & 2 & 0.24 & 0.9981 & 0.9996 & 0.0156 & 0.0455 \\
\hline 6. Egypt & 5.06 & 2 & 0.08 & 0.9925 & 0.9985 & 0.0210 & 0.0877 \\
\hline 7. France & 13.23 & 2 & 0.00 & 0.9681 & 0.9936 & 0.0169 & 0.2047 \\
\hline 8. HK & 5.49 & 2 & 0.06 & 0.9933 & 0.9987 & 0.0151 & 0.0912 \\
\hline 9. Hungary & 11.46 & 2 & 0.00 & 0.9657 & 0.9931 & 0.0140 & 0.2186 \\
\hline 10. Italy & 13.11 & 2 & 0.00 & 0.9793 & 0.9959 & 0.0191 & 0.1654 \\
\hline 11. Macedonia & 13.52 & 2 & 0.00 & 0.9722 & 0.9944 & 0.0382 & 0.1684 \\
\hline 12. Malaysia & 17.00 & 2 & 0.00 & 0.9717 & 0.9943 & 0.0207 & 0.1941 \\
\hline 13. Malta & 25.48 & 2 & 0.00 & 0.9545 & 0.9909 & 0.0178 & 0.2429 \\
\hline 14. Mexico & 4.04 & 2 & 0.13 & 0.9972 & 0.9994 & 0.0087 & 0.0589 \\
\hline 15. Nigeria & 30.86 & 2 & 0.00 & 0.9419 & 0.9884 & 0.0920 & 0.2693 \\
\hline 16. Oman & 40.27 & 2 & 0.00 & 0.9296 & 0.9859 & 0.0370 & 0.3070 \\
\hline 17. Peru & 5.87 & 2 & 0.05 & 0.9919 & 0.9984 & 0.0143 & 0.1012 \\
\hline 18. Philippines & 10.21 & 2 & 0.01 & 0.9842 & 0.9968 & 0.0316 & 0.1436 \\
\hline 19. Portugal & 5.92 & 2 & 0.05 & 0.9921 & 0.9984 & 0.0112 & 0.0992 \\
\hline 20. Romania & 9.11 & 2 & 0.01 & 0.9843 & 0.9969 & 0.0144 & 0.1337 \\
\hline 21. Russia & 5.53 & 2 & 0.06 & 0.9908 & 0.9982 & 0.0235 & 0.0942 \\
\hline 22. Singapore & 2.23 & 2 & 0.33 & 0.9989 & 1.0000 & 0.0063 & 0.0184 \\
\hline 23. Slovenia & 7.33 & 2 & 0.03 & 0.9897 & 0.9979 & 0.0092 & 0.1158 \\
\hline 24. S. Africa & 0.05 & 2 & 0.07 & 1.0000 & 1.0000 & 0.0049 & 0.0000 \\
\hline 25. S. Korea & 5.53 & 2 & 0.06 & 0.9940 & 0.9988 & 0.0089 & 0.0934 \\
\hline 26. Spain & 4.01 & 2 & 0.13 & 0.9957 & 0.9991 & 0.0136 & 0.0743 \\
\hline 27. Taiwan & 2.17 & 2 & 0.34 & 0.9996 & 0.9999 & 0.0102 & 0.0207 \\
\hline 28. Thailand & 5.24 & 2 & 0.07 & 0.9936 & 0.9987 & 0.0243 & 0.0902 \\
\hline 29. USA & 1.82 & 2 & 0.40 & 1.0000 & 1.0000 & 0.0068 & 0.0000 \\
\hline
\end{tabular}

Note: We retained a sample if it satisfied the following four rigorous criteria (i.e. TLI $>0.95$, GFI $>0.95$, SRMSR $<0.08$, RMSEA $<0.08$ ). We eliminated 20 samples (printed in bold).

\section{Step 2: Measurement Invariance of the Pay Level Satisfaction Scale}

Model 1: Configural invariance. We examined the fit between the 4-item, 1-factor PLSS (first-order factor model) and data from each sample and repeated the procedure 29 times (Table 4). On the basis of the four rigorous criteria, we eliminated 20 samples and retained 9 samples. Again, we used EFA to identify the reasons for non-invariance. For instance, for the Nigerian sample, there were two factors for the 4-item PLSS. We combined items 1 and 4 as factor 1 and items 2 and 3 as factor 2 in a modified CFA and set these two factors as related factors (covariance) and found an excellent fit $\left(\chi^{2}=0.02, \mathrm{df}=1, \mathrm{p}=0.88, \mathrm{TLI}=1.0000\right.$, $\mathrm{CFI}=1.0000, \mathrm{SRMSR}=0.0011, \mathrm{RMSEA}=0.0000)$. 
Model 2: Metric invariance. Based on data from nine geopolitical entities ( $\mathrm{N}=2159)$ at the 'scale' level, the difference between the unconstrained MGCFA (Table 3, step 2, model 2A) and the constrained MGCFA (step 2, model 2B) was non-significant based on fit index change $(\Delta \mathrm{CFI}=0.0018)$. We achieved metric invariance for the PLSS.

Models 3 (item-level metric invariance, e.g., item 1), 4 (scalar invariance), and 5 (first-order latent mean comparison) were also examined and presented in Table 3 (step 2). Since all the procedures related models 3 to 5 for the PLSS were all similar to our presentations for the LOMS, we will not present the results in detail here. Results revealed that, for example, the CFI change $(0.0129)$ of scalar invariance was greater than 0.01 but smaller than 0.02 . These minor and potential differences can be further investigated using the same procedure presented in models 3 and 4 of step 1. In summary, among 29 samples, only five samples passed our criteria for both measures. They are Brazil, China, South Africa, Spain and the USA. We now focus on these five samples in subsequent analyses.

\section{Step 3: Common Method Biases Test}

Harman's single-factor test. Common method bias is a potential problem because we collected self-reported data from one source at one point in time. We conducted Harman's one factor test (Podsakoff et al., 2003), examined the unrotated factor solution involving items of all variables of interest (13 items; the 9-item, 3-factor LOMS and the 4-item, 1-factor PLSS) in an exploratory factor analysis (EFA), and found the variance explained to be $29.06 \%, 22.03 \%, 10.39 \%$ and $8.21 \%$ for the four factors, respectively. The first factor covered all items of the LOMS. The second factor had all items of the PLSS. Two additional factors were related to the LOMS with some cross-loadings. No single factor accounted for the majority of the covariance in the data. Thus, common method bias could not account for all of the relationships among the scale items.

Controlling for the effects of a single unmeasured latent method factor. To demonstrate that the results are not due to common method variance, measurement model with the addition of a latent common method variance factor (CMV) must not significantly improve the fit over our measurement model without the latent common method variance factor. With a latent common methods variance factor, 'the variance of the responses to a specific measure is partitioned into three components: (a) trait; (b) method; and (c) random error' (Podsakoff et al., 2003, p. 891). We compared the measurement model without the common methods variance factor (Table 3, step 3 , model 1) with the model with it (model 2) and found that the fit index change was not significant $(\Delta \mathrm{CFI}=0.0009)$. The factor loadings of these items remain significant. On the basis of the results, we may conclude that the method effects are indeed minor and non-significant. 


\section{Step 4: The Functional Equivalence of the Love of Money Scale}

On the basis of results from steps 1 to 3 , we combined these two scales, LOMS and PLSS, into a SEM model and tested for functional equivalence in four separate steps (models) (Table 3, step 4, and Fig. 1). Model 1 was the unconstrained baseline model. When testing functional equivalence, we did not need scalar equivalence (e.g., skipped models 4, 5, and 7 of step 1 for LOMS) for the constrained model but did constrain the gammas (factor loadings) and the betas (the relationships among two endogenous variables) across samples to be equal in three steps.

In model 2, more specifically, we constrained all first-order and second-order factor loadings of the LOMS to be the same across samples (Table 3, step 4) and compared it with the baseline model (model 1). The non-significant CFI change $(0.0011)$ revealed that the LOMS was invariant across samples in this SEM model.

In model 3, we further constrained the first-order factor loadings of the PLSS to be the same across geopolitical entities. The non-significant difference between models 3 and 2 ( $\triangle \mathrm{CFI}=0.0009$ ) suggested that in this SEM model, the PLSS was invariant across samples.

In model 4, we further set the LOMS to PLSS path to be equal across samples. The non-significant CFI change (0.0003) between models 4 and 3 revealed functional equivalence across these five samples. A path is significant at different significance levels $(\mathrm{p}<0.05,0.01,0.001)$ when the critical ratio, C.R., is greater than or equal to $1.96,2.58$ and 3.50, respectively. Standardized regression weights were as listed Brazil $(-0.03$, C.R. $=-0.985)$, China $(-0.05)$, South Africa $(-0.05)$, Spain $(-0.04)$, and the USA $(-0.03)$, respectively. The factor loadings for factors rich, motivator, and important were as follows: Brazil $(0.63,0.56,0.48)$, China $(0.95,0.78,0.72)$, South Africa $(0.69,0.66,0.67)$, Spain $(0.86,0.71,0.78)$, and the USA $(0.88,0.63,0.68)$. Factor rich, again, had the highest factor loading for the LOMS, China (0.95), in particular. Finally, in Model 4, the unstandardized estimates of the regression weight, the standard error, and critical ratio were exactly the same across all five samples. The Love of Money to Pay Level Satisfaction path $(-0.05)$ was non-significant and the factor loadings for LOMS were 1.00 (rich), 0.88 (motivator), and 0.65 (important). In summary, we achieved measurement invariance and functional equivalence for both scales. Among the five samples, the love of money is negatively but non-significantly related to pay level satisfaction.

\section{DISGUSSION}

Both the LOMS and the PLSS were developed by scholars in the USA and have been used in the literature extensively in cross-cultural research. No systematic examination of measurement invariance, however, has been performed in a large number of countries. The present study explored both the LOMS and PLSS in 29 geopolitical entities around the world and provides the following theoretical, empirical and practical contributions to the literature. 
In our theoretical model, the love of money is a second-order latent variable (factor) and is unobservable; that pay level satisfaction is a first-order latent variable (factor) and is also unobservable. The love of money is further defined by three first-order latent variables (factors). The only observable and measurable variables in our model are the nine items of the LOMS and the four items of the PLSS. The first-order factor means are a function of the intercepts of the measured variables and the first-order factor loadings and means. Moreover, the second-order factor mean is a function of the intercepts of the first-order factors, and second-order factor loadings and means (Chen et al., 2005). Therefore, in order to interpret the relationship between love of money and pay level satisfaction, we illustrate the procedures and pass all the measurement invariance/equivalence tests to reach this goal.

In step 1, only 17 samples pass the criteria for the LOMS (12 fail to pass). In step 2, only nine samples pass the criteria for the PLSS (20 fail to pass). Only five samples pass the criteria for both LOMS and PLSS. Results of step 3 reveal the non-significant common method effect. In step 4, we achieve functional equivalence across five samples and identify a negative, but non-significant, relationship between the love of money and pay level satisfaction. We dig deeper in identifying: (i) the specific factor; (ii) the specific item; and (iii) the specific samples at the item level that may contribute to non-invariance. After identifying the non-invariant item(s), researchers can create a partial invariance model that constrains all other items and allows that specific item(s) to vary. We offer the following points.

First, in this study, factor rich, the affective component of the LOMS that shows one's emotions/value-laden orientation, is the most critical component of LOMS. These three items of factor rich may reveal the most important and meaningful cross-cultural differences regarding the love of money. Second, we pay close attention to item 1 ( $I$ want to be rich). When the 'individual self' is the center of the respondents' psychological field for items of a scale ('I' orientation), people in individualistic cultures (Yu and Yang, 1994) may have different perceptions than those in collectivistic cultures (Riordan and Vandenberg, 1994; Tang et al., 2002). We speculate: at the item level, people in high collectivistic cultures (e.g., China, South Korea) may consider 'I want to be rich' not acceptable in their cultures and may have a tendency to display a lower factor loading for the item with the 'I' orientation (see Appendix II, row 1, L: China $=0.833$, South Korea $=0.766$ ) than those in individualistic cultures (e.g., Belgium $=1.471$ ). Third, at the factor level, factor rich has the highest factor loading of three factors for the love of money construct (step 4). In fact, the Chinese sample has the highest factor loading (0.95) for factor rich among these five samples. Future research should explore how national culture may influence perceptions of money across societies.

Four strategies may be used to deal with items that are not metric invariant (the unit of the measurement): (i) ignore the non-invariance because the comparison of 
data is not meaningful; (ii) eliminate non-invariant items from the scale; (iii) invoke partial metric invariance that allows the factor loading of non-invariant items to vary; and (iv) interpret the source of non-invariance (Cheung, 2002). Our experiences suggest that metric non-invariance should not be ignored. Eliminating non-invariance items and/or specific samples may cause the loss of valuable information. Researchers may invoke partial metric invariance (step 1, model 3). Not only is metric non-invariance desirable but also is 'a source of potentially interesting and valuable information about how different groups view the world' (Cheung and Rensvold, 2002, p. 252). In general, our results suggest some possible culture differences in the fine nuances of the meaning of money that should be explored in depth in future research.

\section{Implications for Future Research}

Researchers should not take the measurement invariance of any scales across cultures for granted (Riordan and Vandenberg, 1994). The meanings of money reflect the culture, language, history, people, political systems, social perceptions and the value of the currency in each nation. The relationship between the subject of the research, for example, money, and the extent to which people's personal involvement in responding to the questionnaire in the context of culture, that is, the 'I' orientation, may vary across geopolitical entities. This may have accounted for the low invariance in the item involving the ' $\mathrm{I}$ ' word. This suggests that researchers should examine the wording or phrasing of items carefully when they design future measurement instruments for use in different cultural or national contexts.

CFA is theory-driven. For the PLSS, a sample from Nigeria, for example, fails the configural invariance. Ethnic groups within some samples differ significantly in their history, culture, religion, language, social-economic status and values towards the love of money. For the Nigerian sample, there are many ethnic groups, such as Igbo, Yoruba, Housa and others. Differences in sample composition may explain the fact that Nigeria fails in configural invariance for both the LOMS and the PLSS and may prevent it from having a good fit.

While each measure fits well in many samples, the two measures together fit well in only five samples (including China). Future research may try to control for characteristics that may introduce variance in the understanding or experience of a phenomenon, or identify ways to revise the model. Future research also could explore whether the lack of experience in answering survey questionnaires in several under-represented samples (e.g., Hungary, Macedonia, Malta, Nigeria Oman, etc.) also may contribute to non-invariance.

At the present time, assessment of fit is an active area of research. According to Chen et al. (2005), 'the best available guidelines are probably those proposed by Cheung and Rensvold (2002)' (p. 482). In testing configural invariance for LOMS and PLSS, the majority of our non-invariant samples fail to pass the RMSEA 
among the four criteria. RMSEA is one of the absolute fit indices that assess the degree to which the model implied covariance matrix matches the observed covariance matrix that have a built in penalty for lack of parsimony. RMSEA tends to over-reject a true model when sample sizes are small and is more likely to be affected by sample size and model complexity. The small size in many samples of this study, close to 200, may be one of the causes for non-invariance. Researchers may explore similar or different values for indices (e.g., CFI, SRMSR and RMSEA) in testing different invariance (e.g., loading, intercept and residual invariance) and use their sound judgment and substantive expertise in making decisions (Chen, in press). Cleary, more research is needed in this direction.

The lack of an empirical relationship between the love of money and pay level satisfaction in these five samples suggests the possibility of potential moderators that may either attenuate or enhance the relationship. Are there moderators that could be introduced into the future theorizing and research on the nature of the relationship between love of money and other attitudinal or behavioural responses? Our rigorous criteria significantly reduce the number of samples eligible for subsequent data analyses (model 1 for steps 1 and 2) that may contribute to our findings. More research is needed to identify measures with theoretical importance and measurement and functional equivalence in management and organization research.

Lastly, this LOMS has passed the measurement invariance test as well as the functional equivalence test in the Chinese sample. This may contribute to future studies on the role of money for organizational behaviour within the Chinese context. A key issue in doing business in China is 'corruption'. The love of money may be the underlying motive for corrupt behaviour. China is ranked $57^{\text {th }}$ on the Corruption Perception Index (http://www.transparency.org/documents/cpi/ 2001/cpi2001.html). At the same time, Chinese people, relatively speaking, have low income levels (GDP per capita in $2004=\$ 5600$ ). With all the economic changes, the importance of money and the love of money also may be very interesting social and psychological phenomena. Does love of money contribute to corrupt behaviour? Future research could correlate the love of money scale with corruption indices across countries. Does love of money motivate productive behaviour at the individual level and economic growth at the firm or national level? The love of money may play a role in our understanding of people's work-related attitudes and behaviours in the emerging world markets, for example, job satisfaction, turnover, helping behaviour and unethical behaviour in China in particular. It is a human resources management issue at both the firm and the national levels.

\section{Limitations}

We do not have control over many extraneous or nuisance variables that may introduce bias into the responses (e.g., the size of the organization, organizational 
culture, economy of the nation/region, unemployment rate, and participants' knowledge of the English language, management literature, and the purpose of this research project). Extraneous variables are potential independent variables that could exert a systematic influence on the measurements in a study. However, with 29 geopolitical entities, these extraneous variables are distributed randomly and may not have a systematic impact on the results of this study. A second limitation is that the convenience samples drawn from each society are small and may not represent the average citizen of the geopolitical entity. It is plausible that with adequate sample size $(\mathrm{N}>300)$; we may have different patterns of results.

\section{GONCLUSION}

This paper provides a detailed procedure to evaluate the measurement and functional equivalence of a construct for cross-cultural research. In this process, we suggest several methods for identifying the sources of invariance and strategies for dealing with the lack of invariance. We hope that this paper contributes to the overall goal of developing valid measures for cross-cultural management research in general and to Chinese management research in specific.

\section{NOTES}

Portions of this paper were presented at the $63^{\text {rd }}$ Academy of Management Annual Meeting, Seattle, WA, August 3-6, 2003. The authors would like to thank the editor-in-chief, Anne S. Tsui, Gordon W. Cheung, Fang Fang Chen, and two reviewers for their insightful and constructive comments on earlier versions of this paper, the Faculty Research and Creative Activity Committee of MTSU for financial support, the late Father Wiatt A. Funk for his suggestions, and James Weston Van Buren and Grant Hofmann for their assistance. The senior author would like to dedicate this research (his $100^{\text {th }}$ journal article) to his parents, the late Kuan-Ying Tang (汤冠英), one of the four founding fathers of the Department of Psychology at National Taiwan University, and the late Fang Chen Chu Tang (汤朱芳貞). Address all correspondence to Thomas Li-Ping Tang. 


\section{APPENDIX I}

\section{Items of the Love of Money Scale and Pay Level Satisfaction Scale}

The Love of Money Scale

Factor rich

1. I want to be rich.

2. It would be nice to be rich.

3. Have a lot of money (being rich) is good.

Factor motivator

4. I am motivated to work hard for money.

5. Money reinforces me to work harder.

6. I am highly motivated by money.

Factor important

7. Money is good.

8. Money is important.

9. Money is valuable.

Response scale (1) strongly disagree, (3) neutral, and (5) strongly agree.

Pay Level Satisfaction Scale

1. My take-home pay

2. My current salary

3. My overall level of pay

4. Size of my current salary

Response scale: (1) strongly dissatisfied, (3) neutral, and (5) strongly satisfied.

The Chinese version of the scales is available on $M O R$ website: www.iacmr.org and also from the first author of this article. 


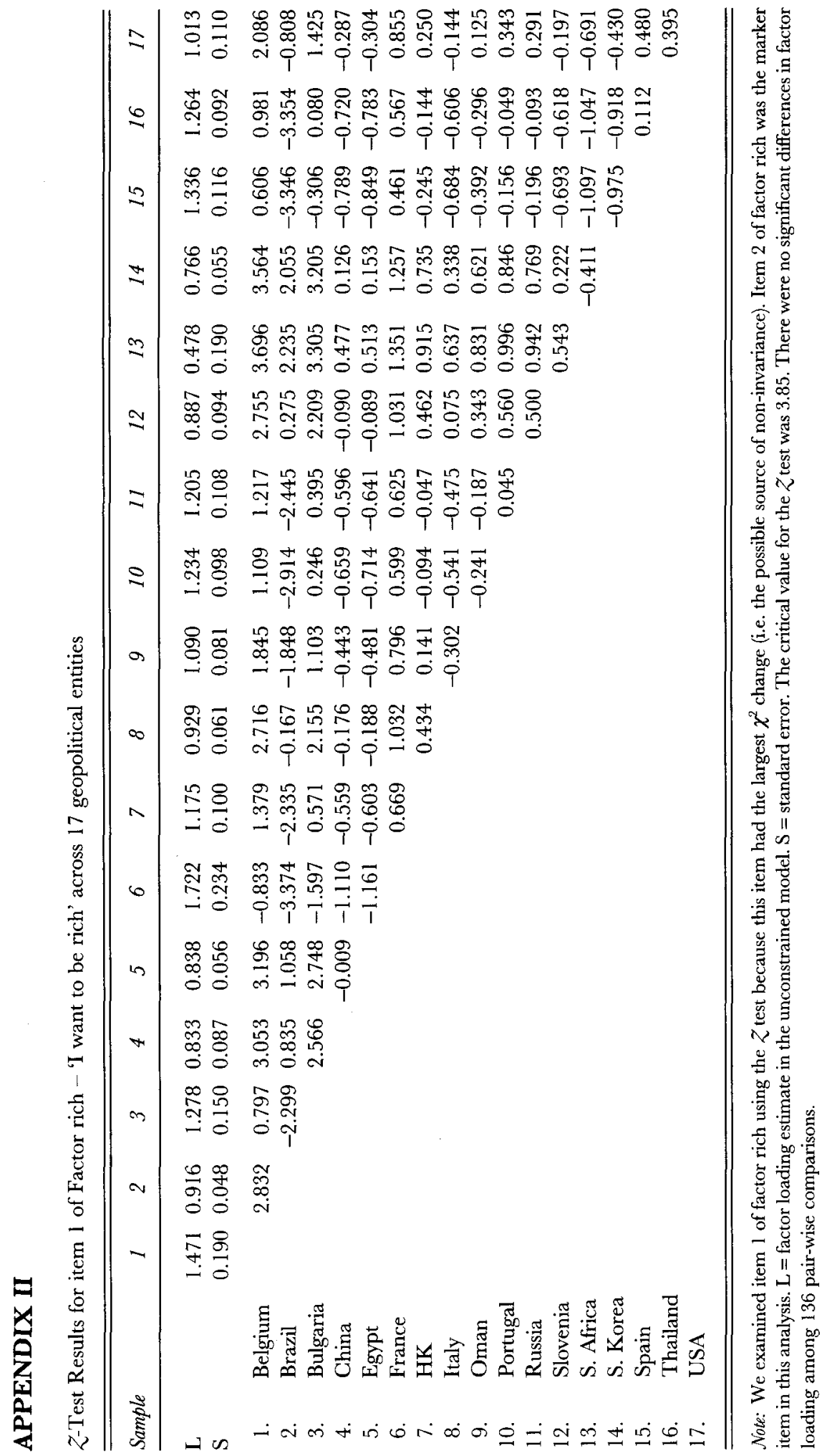




\section{REFERENGES}

Adams, J. (1963). 'Toward an understanding of inequity'. Fournal of Abnormal Social Psychology, 67, $422-36$.

Bandalos, D. L., and Finney, S. J. (2001). 'Item parceling issues in structural equation modeling'. In Marcoulides, G. A. (Ed.), New Developments and Techniques in Structural Equation Modeling. Mahwah, NJ: Lawrence Erlbaum Associates, (pp. 269-96).

Belk, R. W. (1985). 'Materialism: Trait aspects of living in the material world'. Journal of Consumer Research, 12, 265-80.

Boyacigiller, N., and Adler, N. J. (1991). 'The parochial dinosaur: Organizational science in a global context'. Academy of Management Review, 16, 262-90.

Brislin, R. W. (1980). 'Translation and content analysis of oral and written materials'. In Triandis, H. C., and Berry, J. W. (Eds), Handbook of Cross-Cultural Psychology, vol. 2: Methodology. Boston, MA: Allyn and Bacon, (pp. 349-444).

Bruner, J. S., and Goodman, C. C. (1947). 'Value and needs as organizing factors in perception'. Fournal of Abnormal and Social Psychology, 42, 33-44.

Bryan, R. G. (2004). 'A survey on causes of dissatisfaction at college'. Paper presented at the Annual Convention of the American Psychological Association, July 28-August 1, Honolulu, HI.

Chen, F. F. (in press). 'Sensitivity of goodness of fit indices to lack of measurement invariance'. Structural Equation Modeling.

Chen, F. F., Sousa, K. H., and West, S. G. (2005). 'Testing measurement invariance of second-order factor models'. Structural Equation Modeling, 12, 3, 471-92.

Chen, Y. J., and Tang, T. L. P. (2006). 'Attitude toward and propensity to engage in unethical behavior: Measurement invariance across major among university students'. Foumal of Business Ethics (Published online: 5 July 2006, DOI: 10.1007/s10551-006-9069-6).

Cheung, G. W. (2002). 'The identification of a partial metric invariance model for multi-group studies'. Paper presented at the Academy of Management Annual Meeting, August, 11-14, Denver, CO.

Cheung, G. W., and Rensvold, R. B. (2002). 'Evaluating goodness-of-fit indexes for testing measurement invariance'. Structural Equation. Modeling, 9, 2, 233-55.

Chiu, R. K., Luk, V., and Tang, T. L. P. (2001). 'Hong Kong and China: The cash mentality revisited'. Compensation and Benefits Review, 33, 3, 66-72.

Cohen-Charash, Y., and Spector, P. E. (2001). 'The role of justice in organizations: A meta-analysis'. Organizational Behavior and Human Decision Processes, 86, 278-321.

Du, L. Z., and Tang, T. L. P. (2005). 'Measurement invariance across gender and major: The Love of Money among university students in People's Republic of China'. Fournal of Business Ethics, 59, 281-93.

Furnham, A., and Argyle, M. (1998). The Psychology of Money. London: Routledge.

Harpaz, I. (1990). 'The importance of work goals: An international perspective'. Fournal of International Business Studies, 21, 1, 79-93.

Heneman, H., and Schwab, D. (1985). 'Pay satisfaction: Its multidimensional nature and measurement'. International Journal of Psychology, 20, 129-41.

Heneman, H. G., and Judge, T. A. (2000). 'Compensation attitudes'. In Rynes, S. L., and Gerhart, B. (Eds), Compensation in Organizations: Current Research and Practice. San Francisco, CA: Jossey-Bass, (pp. 61-103).

Herzberg, F. (1987). 'One more time: How do you motivate employees?'. Harvard Business Review, September-October, 109-120 (Originally published January-February 1968).

Hofstede, G. (1980). 'Motivation, leadership, and organization: Do American theories apply abroad?'. Organizational Dynamics, $9\langle 1\rangle, 42-63$.

Hom, P. W., and Griffeth, R. W. (1995). Employee Tumover. Cincinnati, OH: South-Western College Publishing.

Hu, L., and Bentler, P. M. (1999). 'Cutoff criteria for fit indexes in covariance structure analysis: Conventional criteria versus new alternatives'. Structural Equation Modeling, 6, 1-55.

Jurgensen, C. E. (1978). 'Job preferences (what makes a job good or bad?)'. Journal of Applied Psychology, 63, 267-76.

Kohn, A. (1993). 'Why incentive plans cannot work'. Harvard Business Review, September/October, 5463. 
Law, K. S., Wong, C. S., and Mobley, W. H. (1998). 'Toward a taxonomy of multidimensional constructs'. Academy of Management Review, 23, 4, 741-55.

Lawler, E. E. (1971). Pay and Organizational Effectiveness: A Psychological Viere. New York: McGraw-Hill.

Lim, V. K. G., and Teo, T. S. H. (1997). 'Sex, money and financial hardship: An empirical study of attitudes towards money among undergraduates in Singapore'. Fournal of Economic Psychology, 18, $369-86$.

Locke, E. A. (1969). 'What is job satisfaction?'. Organizational Behavior and Human Performance, 4, 309-36.

Locke, E. A. (1976). 'The nature and causes of job satisfaction'. In Dunnette, M. D. (Ed.) Handbook of Industrial and Organizational Psychology. Chicago: Rand McNally College Publishing Company, (pp. 1297-1349).

Locke, E. A., Feren, D. B., McCaleb, V. M., Shaw, K. N., and Denny, A. T. (1980). 'The relative effectiveness of four methods of motivating employee performance'. In Duncan, K, D., Gruneberg, M. M., and Wallis, D. (Eds), Changes in Working Life. New York: Wiley, (pp. 363-88).

Luna-Arocas, R., and Tang, T. L. P. (2004). 'The love of money, satisfaction, and the Protestant Work Ethic: Money profiles among university professors in the USA and Spain'. Joumal of Business Ethics, 50, 329-54.

Milkovich, G. T., and Newman, J. M. (2005). Compensation, 8th edn. Boston, MA: Irwin/McGrawHill.

Mitchell, T. R., and Mickel, A. E. (1999). 'The meaning of money: An individual difference perspective'. Academy of Management Review, 24, 3, 568-78.

Opsahl, R. L., and Dunnette, M. D. (1966). 'The role of financial compensation in industrial motivation'. Psychological Bulletin, 66, 94-118.

Pfeffer, J. (1998). 'Six dangerous myths about pay'. Harvard Business Reviewe, May/June, 108-19.

Podsakoff, P. M., MacKenzie, S. B., Lee, J. Y., and Podsakoff, N. P. (2003). 'Common method biases in behavioral research: A critical review of the literature and recommended remedies'. Journal of Applied Psychology, 88, 879-903.

Rice, R. W., Phillips, S. M., and McFarlin, D. B. (1990). 'Multiple discrepancies and pay satisfaction'. Fournal of Applied Psychology, 75, 4, 386-93.

Riordan, C. M., and Vandenberg, R. J. (1994). 'A central question in cross-cultural research: Do employees of different cultures interpret work-related measures in an equivalent manner?'. Journal of Management, 20, 643-71.

Samiee, S., and Jeong, I. (1994). 'Cross-cultural research in advertising: An assessment of methodologies'. Joumal of the Academy of Marketing Science, 22, 3, 205-17.

Sin, L. Y. M., Cheung, G. W. H., and Lee, R. (1999). 'Methodology in cross-cultural consumer research: A review and critical assessment'. Fournal of International Consumer Marketing, 11, 4, 75-96.

Smith, A. (1937). An Inquiry into the Nature and Causes of the Wealth of Nations. New York: Modern Library (Original work published 1776).

Stajkovic, A. D., and Luthans, F. (2001). 'Differential effects of incentive motivators on work performance'. Academy of Management foumal, 44, 580-90.

Tang, T. L. P. (1992). 'The meaning of money revisited'. Journal of Organizational Behavior, 13, 197-202.

Tang, T. L. P. (in press). 'Income and quality of life: Does the love of money make a difference?'. Foumal of Business Ethics.

Tang, T. L. P., and Chiu, R. K. (2003). 'Income, Money Ethic, pay satisfaction, commitment, and unethical behavior: Is the love of money the root of evil for Hong Kong employees?'. Joumal of Business Ethics, 46, 13-30.

Tang, T. L. P., Kim, J. K., and Tang, D. S. H. (2000). 'Does attitude toward money moderate the relationship between intrinsic job satisfaction and voluntary turnover?'. Human Relations, 53, 2, $213-45$.

Tang, T. L., Furnham, A., and Davis, G. M. T. W. (2002). 'The meaning of money: The Money Ethic cndorsement and work-related attitudes in Taiwan, the United States, and the United Kingdom'. Fournal of Managerial Psychology, 17, 7, 542-63.

Tang, T. L., Luna-Arocas, R., and Sutarso, T. (2005). 'From income to pay satisfaction: The love of money and pay equity comparison as mediators and culture (the US and Spain) and gender as moderators'. Management Research: The Fournal of the Iberoamerican Academy of Management, 3, 1, $7-26$. 
The American Freshman (1994). Higher Education Research Institute. Los Angeles, CA: University of California.

Vandenberg, R. J., and Lance, C. E. (2000). 'A review and synthesis of the measurement invariance literature: Suggestions, practices, and recommendations for organizational research'. Organizational Research Methods, 3, 1, 4-69.

Vanneman, R. D., and Pettigrew, T. (1972). 'Race and relative deprivation in the urban United States'. Race, 13, 461-86.

Wernimont, P. F., and Fitzpatrick, S. (1972). 'The meaning of money'. Foumal of Applied Psychology, 56, 218-26.

Williams, M. L., McDaniel, M. A., and Nguyen, N. T. (2006). 'A meta-analysis of the antecedents and consequences of pay level satisfaction'. Journal of Applied Psychology, 91, 392-413.

$\mathrm{Yu}, \mathrm{A}$. B., and Yang, K. S. (1994). 'The nature of achievement motivation in collectivist societies'. In Kim, U., Triandis, H. C., Kagitcibasi, C., Choi, S. C., and Yoon, G. (Eds), Individualism and Collectivism: Theory, Method, and Applications. Thousand Oaks, CA: Sage Publications, (pp. 239-66).

Thomas Li-Ping Tang (ttang@mtsu.edu) is a Full Professor of Management in the Department of Management and Marketing, Jennings A. Jones College of Business at Middle Tennessee State University (MTSU). He received his $\mathrm{Ph} . \mathrm{D}$. in Industrial and Organizational Psychology from Case Western Reserve University. He has taught I/O Psychology at National Taiwan University. Professor Tang's research interest is related to the love of money, pay satisfaction and cross-cultural issues.

Toto Sutarso (tsutarso@mtsu.edu) is a Statistical Research Consultant, Information Technology Division at Middle Tennessee State University. He received his Ph.D. in Applied Statistics and Research Methodology from the University of Alabama. His research interest is centred on attitude measurement, satisfaction, missing data, simulation study, business emotional intelligence, group differences and cross-cultural issues.

Biographies of all other co-authors are available on Thomas Tang's website: www.mtsu.edu/ ttang.

Manuscript received: August 1, 2005

Final version accepted: July 26, 2006

Accepted by: Anne S. Tsui 